\title{
Use of inducible class II and MHC class I-related chain A/B expression on $T$ lymphocytes as an in vitro screen for the immunomodulatory properties of dietary products
}

\author{
C. Carter, K. Varley and B. Clark \\ Transplant and Cellular Immunology, St James' University Hospital, Beckett Street, Leeds LS9 7TF, UK
}

Immune-mediated rejection following renal transplantation is essentially centred around two primary mechanisms. The presence of preformed antibodies against donor human leucocyte antigen (HLA) in sensitised recipients results in hyperacute rejection. Non-self HLA can also trigger a cell-mediated immune response in which a key event is the direct activation of CD4 + T-cells by non-self HLA class II molecules expressed on antigen-presenting cells present in the donor organ. Furthermore, recent reports have highlighted an association between increased MHC class I-related chain A/B (MICA/B) expression in graft tissue and immunological tissue damage and rejection following renal transplantation ${ }^{(1)}$. Protocols that decrease the expression of MICA/B or class II HLA proteins either pre- or post transplantation would be a potentially useful approach in a live donor setting. Whilst considerable efforts are made to control the recipient's immune system post transplant little attention is paid to this aspect in the donor pre-transplant. There are, however, a number of reports suggesting that pharmacological agents (e.g. statins) $)^{(2)}$ as well as dietary products (most notably fish oils) ${ }^{(3)}$ reduce the expression of HLA class II expression. Whilst administering statins to healthy donors before the transplant may be difficult to justify, the use of dietary products such as fish oils may be both beneficial and appropriate. The aim of the present study was to develop an in vitro assay in which the effects of potential immunomodulators on MICA/B and HLA-DR expression were measured. To this end, human T lymphocytes were stimulated with phytohaemagglutinin (PHA) and HLA-DR and MICA/B expression were measured by antibody staining and two-colour flow cytometry. Incubation of peripheral blood mononuclear cells with PHA for 3-5 d led to consistently increased HLA-DR expression. This was found to be the case both in the frequency (\% positive) of CD3 ${ }^{+}$T-cells expressing HLA-DR and in the mean channel fluorescence (MCF) that estimates antigen density (Table). The expression of MICA/B following PHA stimulation was found to be considerably lower than HLA-DR and was considered to be too inconsistent for further consideration in this assay (Table). The kinetics of expression and the required concentration of PHA to give optimal expression as well as alternative stimulators (anti-CD3 stimulation) were also investigated. This in vitro system is now being used to assess the ability of dietary products to down regulate the expression of class II HLA-DR expression on T-cells following stimulation.

Table. Expression of HLA-DR and MICA/B on T-cells following in vitro PHA stimulation

\begin{tabular}{lccccr}
\hline & \multicolumn{2}{c}{ CD3/HLA-DR } & & \multicolumn{2}{c}{ CD3/MICA/B } \\
\cline { 2 - 3 } \cline { 6 - 7 } & $\%$ Positive & MCF & & Positive & MCF \\
\hline Unstimulated & 8.62 & 1.32 & & 0.04 & 3.05 \\
+ PHA & 64 & 407 & & 16.4 & 8.00 \\
\hline
\end{tabular}

1. Hankey KG, Drachenberg CB, Papadimitriou JC, Klassen DK, Philosophe B, Bartlett ST, Groh V, Spies T \& Mann DL (2002). Transplantation 73, 304-306.

2. Kuipers HF, Biesta PJ, Groothuis TA, Neefjes JJ, Mommaas AM \& van den Elsen PJ (2005) Hum Immunol 66, 653-665.

3. Hughes DA, Pinder AC, Piper Z, Johnson IT \& Lund EK (2000) Am J Clin Nutr 71, Suppl., 343S-348S. 